\title{
A BI-LEVEL FRAMEWORK FOR PRICING OF HIGH-OCCUPANCY TOLL LANES
}

\author{
Kitae Jang ${ }^{1}$, Myoung Kyun Song ${ }^{2}$, Keechoo $\mathrm{Choi}^{3}$, Dong-Kyu Kim ${ }^{4}$ \\ ${ }^{1}$ The Cho Chun Shik Graduate School for Green Transportation, \\ Korea Advanced Institute of Science and Technology, South Korea \\ ${ }^{2}$ Small and Medium Business Office Marketing Team, SK Telecom, South Korea \\ ${ }^{3}$ Dept of Transportation Systems Engineering, Ajou University, South Korea \\ ${ }^{4}$ Dept of Civil and Environmental Engineering, Seoul National University, South Korea
}

Submitted 3 December 2013; resubmitted 27 February 2014; accepted 16 June 2014

\begin{abstract}
As a freeway operational management strategy, High-Occupancy Toll (HOT) lanes have been deployed to manage the demand for High-Occupancy Vehicle (HOV) lanes by adjusting the tolls. By doing so, the capacity of freeways with such lanes can be used more efficiently. Periodically, setting the right amount of toll in accordance with the time-varying demand is a key to successful operation of HOT lanes; however, this is often difficult because travellers have heterogeneous willingness to pay for the toll and traffic conditions vary as the demand changes due to the imposition of tolls. This paper proposed an algorithm to determine the optimal level of toll for minimizing the total delay collectively spent by both HOVs and low-occupancy vehicles. Based on real-world traffic and survey data obtained from Gyungbu expressway in South Korea, a case study is presented to verify the applicability of the developed algorithm. The results from the case study show that the proactive dynamic pricing scheme can use the underutilized capacity of HOT lane efficiently and, thereby, shorten total travel time by $22 \%$ and generate revenue of more than $\$ 8600$. Some limitations and future research agendas are also discussed.
\end{abstract}

Keywords: heterogeneous drivers; congestion pricing; high-occupancy toll lane; delay minimization; willingness to pay. Reference to this paper should be made as follows: Jang, K.; Song, M. K; Choi, K.; Kim, D.-K. 2014. A bi-level framework for pricing of high-occupancy toll lanes, Transport 29(3): 317-325. http://dx.doi.org/10.3846/16484142.2014.952248

\section{Introduction}

Managed lanes, especially High-Occupancy Vehicle (HOV) lanes, have been implemented extensively on congested urban freeways in an effort to encourage solo drivers to carpool or use other shared transportation modes, thereby diminishing vehicular demand. The diminished demand is expected to mitigate congestion, and also reduce energy consumption and pollutant emissions (Boriboonsomsin, Barth 2007; Burris, Lipnicky 2009; Mannering, Hamed 1990; Small et al. 2006). Since the mid-90s, however, there have been criticisms of these facilities, including the underused capacity of the HOV lanes (often called empty lane syndrome) and the unfairness of using those lanes (Burris, Appiah 2004; Chen, Varaiya 2005; Dahlgren 1998; Kwon, Varaiya 2008; Li et al. 2007).

To address the aforementioned issues, the HighOccupancy Toll (HOT) concept was introduced. HOT facilities can make better use of the underutilized capac- ity of HOV lanes by allowing the drivers of Low-Occupancy Vehicle (LOVs) to use HOV lanes for a fee. This road-pricing scheme also generates revenue that can be invested in improving the roadways (Poole 2000; Burris, Stockton 2004). Due to these advantages, transportation agencies in many countries, including South Korea, have considered deploying HOT lanes on their freeway systems. Successful implementation of HOT lanes requires an efficient pricing strategy that can realistically reflect conditions on freeways with HOT lanes, especially timevarying traffic conditions and drivers' heterogeneous behaviours in deciding whether, when, and to what extent to use HOT lanes.

Many studies (Brownstone, Small 2005; Chung, Choi 2010; De Palma, Lindsey 2011; Supernak et al. 2003; Yin, Lou 2009) have proposed various dynamic pricing algorithms that periodically adjust toll amounts to be compatible with varying traffic conditions, i.e., adjusting toll price as functions of demand for General

Corresponding author: Dong-Kyu Kim

E-mail: dongkyu@snu.ac.kr 
Purpose (GP) lanes and utilization level in HOT lanes. However, these studies assumed that all drivers have the same Willingness To Pay (WTP) for saving travel time. This assumption was unrealistic because drivers are known to exhibit heterogeneous perceptions even for the same amount of travel time (Amador et al. 2005; Brownstone et al. 2003; Hensher, Greene 2011), such that their choices of which lanes to use, i.e., whether they pay to travel in the HOT lanes, can be different. Some studies (De Palma, Lindsey 2004; Jang et al. 2014; Small, Yan 2001) considered heterogeneity in travellers' WTP in developing HOT pricing strategies, and, by realistically replicating drivers' behaviours in choosing which lanes to use, they found that the strategies that were developed resulted in different outcomes.

Furthermore, the pricing algorithms proposed in the above studies only reflected travel times at the moment that the drivers entered the HOV lanes; however, as the toll price is imposed, some LOVs change their lanes from GP to HOT lanes, thereby reducing the demand for GP lanes. Thus, the difference in travel times between HOT and GP lanes decreases because drivers in the GP lanes can travel faster as the smaller number of vehicles uses GP lanes. Hence, the actual travel times differ from the expected ones at the moment they chose to use the HOT lane. This means that, in order to provide more accurate information for drivers to make right decisions, the HOT pricing algorithm must reflect varying traffic conditions more accurately.

The objective of this study is to develop a HOT pricing algorithm that reflects heterogeneous travellers' WTP and that can proactively adjust toll prices to match traffic conditions. The remainder of this paper is organized as follows. Section 1 explains the pricing algorithm that was developed. Section 2 describes computational simulations performed to demonstrate the applicability of the algorithm and documents findings. Latest section provides a summary of our results and suggestions for future research.

\section{Pricing Algorithm}

Toll pricing strategies for operating HOT lanes are often unable to achieve the optimal performance of the transportation system unless the toll prices they impose properly and continuously reflect drivers' WTP for travel time savings and travel conditions. The pricing algorithm described in this section was developed to take into account drivers' heterogeneous WTP for travel time savings as well as varying travel conditions. The distribution of the former was estimated by using a mixed logit model (Section 1.1). Then, the distribution was used to determine the instantaneous, optimal toll prices in accordance with the difference in travel time for the GP and HOT lanes (Section 1.2). The imposition of toll price induces LOVs to alter their lanes from GP to HOT lanes. This change in demand also affects travel time (Section 1.3). The present study uses a bilevel pricing framework: at the upper-level, the toll price is determined based on input demand and travel time; at the lower-level, demand and travel time are updated by proactively reflecting drivers' responses to the toll price determined at the upper-level. The updated demand and travel time are input again to the upper level. This repetitive process continues until convergence (Section 1.4).

\subsection{Drivers' Willingness to Pay (WTP) for Travel Time Savings}

In the present pricing algorithm, the distribution of drivers' WTP for travel time savings was estimated using a mixed logit model based on the travel survey data. The mixed logit model can obviate the limitations of the standard logit model by allowing for drivers' heterogeneity, which represents the drivers' behaviours concerning lane choices more realistically (Train 2009).

Drivers on freeways with HOT lanes have two alternatives - i.e., HOT and GP lanes - and they make their decisions based on the different attributes of the two alternatives, which are travel time and toll price. Since drivers are already on the freeway and make choices between HOT and GP lanes, it is assumed that other factors are invariant. To replicate and simplify this situation, therefore, survey respondents were asked to choose one of two alternatives that were described by the representative travel attributes of travel time and toll price. The utility of a driver $n$ by choosing alternative $j$ is specified as:

$$
u_{n j}=\beta_{n}^{T} \cdot T_{n j}+\beta_{n}^{C} \cdot C_{n j}+\varepsilon_{n j},
$$

where: $x_{n j}$ is a vector of travel condition attributes of alternative $j$ faced by driver $n ; \beta_{n}^{T}, \beta_{n}^{C}$ are coefficients of travel time and toll price for a driver $n$, respectively, and these coefficients are assumed to be a random term that is an independent, identically-distributed, extreme value.

Since the coefficients, $\beta^{T}$ and $\beta^{C}$, in equation (1) are assumed to vary among drivers with probability density, $f(\beta)$, mixed logit probability can be derived as the integral of standard logit probability over density $f(\beta)$. These coefficients represent quantified relations between drivers' utility and the corresponding variables. The units of $\beta^{T}$ and $\beta^{C}$ are time ${ }^{-1}$ and $\operatorname{cost}^{-1}$, respectively. Therefore, the probability density function of WTP for travel time savings, $w(\times)$, can be computed by taking the ratio of two distributions of time and cost coefficients.

Both $\beta^{T}$ and $\beta^{C}$ have negative values because drivers' utility diminishes as both variables increase. Lognormal distributions are used for those coefficients for the following reasons: (1) comparing with other distributions, it is more convenient to estimate drivers' WTP, which is the ratio of $\beta^{T}$ to $\beta^{C}$, because the ratio of two log-normal distributions also follows a log-normal distribution; and (2) log-normal distribution is single-tailed and limited to one domain and, therefore, it is reported that the log-normal distribution is suitable to represent those coefficients (Hensher, Greene 2003; Hess et al. 2005, Train, Sonnier 2005).

These coefficients can be estimated by using the maximum simulated likelihood estimation method (for 
more details in Hensher et al. 2005a, 2005b; Train 2009). In this study, the Halton sequence was used to draw 1000 random numbers. Since only two variables were included in the model, this method was able to estimate the time and cost coefficients with accuracy and efficiency as reported in previous studies (Bhat 2001; Hensher 2001; Train 1999).

By estimating $\beta^{T}$ and $\beta^{C}$, the cumulative distribution function of WTP, $W(\times)$, can also be derived, meaning that we can now compute a proportion of travellers whose WTP for travel time is greater than a certain level. By taking the inverse of $W(\times)$, therefore, we can also compute what proportion of travellers would respond to the posted toll price, $P$.

\subsection{Toll Price Determination}

For HOT lanes, toll price can be determined for different objectives, such as the minimization of delay and the maximization of revenue (Jang et al. 2014). In the present study, the algorithm was developed to minimize the total travel time that was collectively spent by all travellers on the freeway with the constraints that the traffic in the HOT lane should maintain their speed at the free-flow rate.

$$
\begin{aligned}
& \min \left(T_{H O T} \cdot\left(D_{H O T}^{H O V}+D_{H O T}^{L O V}\right)+T_{G P} \cdot D_{G P}^{L O V}\right) ; \\
& \text { s.t. } D_{H O T}^{H O V}+D_{H O T}^{L O V}+D_{G P}^{L O V}=D ; \\
& v_{H O T}=\text { free-flow speed, }
\end{aligned}
$$

where: $T$ is travel time, $D$ is demand, and $v$ is travel speed. Superscripts indicate demand group, i.e., HOV and LOV, while the subscripts indicate the type of lane that the travellers are using, i.e., HOT and GP lanes.

In this study, it is assumed that the only difference from the HOV facilities is tolling for LOVs (LOVs can access the HOT lane for a fee, while HOVs can use the lane for free.) Among the variables in equation (2), HOV demand, $D_{H O T}^{H O V}$, and LOV demand, $D_{G P}^{L O V}+D_{H O T}^{L O V}$, are determined. The given constraints are that $T_{H O T}$ is always free-flow trip time, and shorter travel time than $T_{G P}$. Therefore, the solution for equation (2) is to maximize $D_{H O T}^{L O V}$ (i.e., allow as many LOVs as possible in the HOT lane) as long as the vehicles in the HOT lane maintain free-flow speed. The solution can be expressed as the minimum value between unused capacity of the HOT lane and the total number of queued vehicles (speed in the HOT lane is assumed to maintain a constant, freeflow speed until the demand reaches the capacity based on the empirical evidence in previous research (Cassidy 1998; Windover, Cassidy 2001)):

$D_{H O T^{*}}^{L O V}=\min \left\{C_{H O V}-D_{H O T}^{H O V}, Q_{G P}+D^{L O V}-C_{G P}\right\}$,

where: $C_{H O V}$ is the capacity of the HOT lane; $Q_{G P}$ is the number of queued vehicles in the GP lanes; $D^{L O V}$ is the LOV demand (which is equal to $D_{G P}^{L O V}+D_{H O T}^{L O V}$ ); $C_{G P}$ is the capacity of the GP lanes.

Now, we know the extent to which LOV demand should be shifted from GP to HOT lanes in order to minimize total delay, and we must determine the price that can attract that much demand, i.e., $D_{H O T^{*}}^{L O V}$, from the GP lanes. Since drivers whose WTP for travel time savings is greater than the imposed toll would shift to the HOT lane, we could compute the price by using $W^{-1}(\times)$, which is the inverse of the distribution of WTP, and, thus, the value of its independent variable is between 0 and 1 . The desired proportion of demand for shift, $D_{H O T^{*}}^{L O V} / D^{L O V}$ is input to $W^{-1}(\times)$ to determine price. Hence, the toll price $P$, is:

$$
P=W^{-1} \cdot\left(1-\frac{D_{H O T^{*}}^{L O V}}{D^{L O V}}\right) \cdot\left(T_{G P}-T_{H O T}\right) .
$$

However, traffic conditions are distorted by the shifted demand from GP to HOT lanes as a result of paying the toll. The varying traffic conditions, demand and travel time, due to the imposition of the toll should be incorporated in the pricing algorithm. The following section describes an approach to updating demand and travel time inputs to the pricing algorithm described in this section.

\subsection{Drivers' Responses and Their Effects}

As drivers respond to the imposition of the toll, demand shift from GP to HOT lanes occurs and, consequently, underused capacity and travel time savings change. This situation can be viewed as a game between drivers and the freeway operator, because the operator decides the optimal toll price by considering drivers' behaviours, and drivers choose their lanes, either HOT lane or GP lanes, according to the toll price determined by the operator. Since the operator already knows drivers' strategy due to the estimated drivers' WTP for travel time savings, the game can be categorized as a Stackelberg game in which the leader knows how the followers will respond to any decision the leader may make (Fisk 1984; Zhang, Ge 2004).

Since drivers respond to the posted toll price, $P$, we need to know how many LOVs choose to travel the HOT lane at the posted price. To this end, the Monte Carlo simulation approach is used to evaluate each LOV driver's response to the posted price as follows:

Step 0: Initial $P$ is computed by equation (4) and set $r=1$ where $r$ is the number of vehicle arrivals.

Step 1: Draw random number $n$ from 0 to 1 , and label $n^{r}$. Step 2: $r$ th LOV chooses to use the HOT lane if their WTP is greater than $P$. Therefore, $D^{L O V}=$ $D^{L O V}-1$, if $W^{-1}\left(n^{r}\right) \geq P ; D^{L O V}=D^{L O V}$; otherwise, go to Step 4 .

Step 3: The change in $D^{L O V}$ diminishes travel time in GP lanes. $T_{G P}$ is re-estimated.

Step 4: Set $r=r+1$, and repeat this procedure (Steps 1, 2, and 3) until $r=D^{L O V}$.

Note that $T_{\mathrm{GP}}$ can be estimated by using a cumulative input-output diagram (Fig. 1). The arrival curve (bold, dark line) is the demand for GP lanes prior to the imposition of the toll, and the slope of departure curve (bold, grey line) represents the departure rate by GP lanes. 


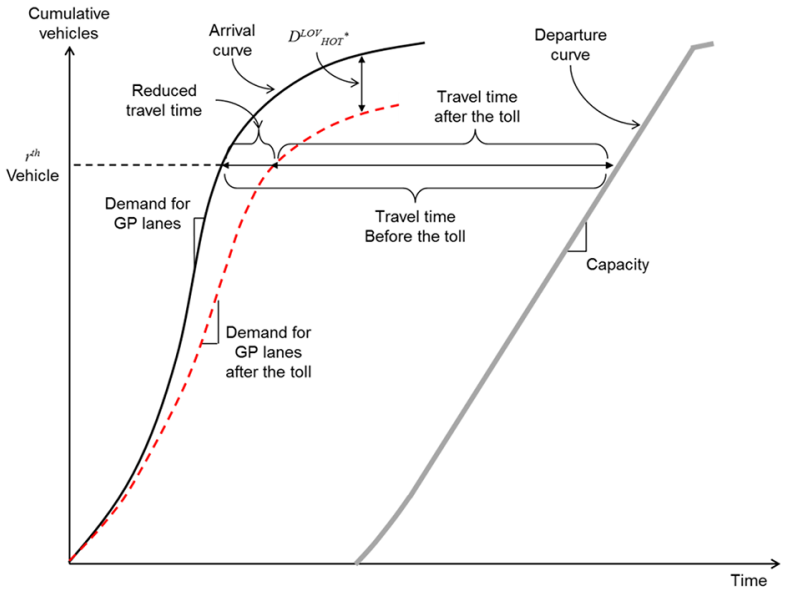

Fig. 1. Input-output diagram for GP lanes

The horizontal distance between the arrival and the departure curves is the total travel time. The amount of demand that shifts from the GP lanes to the HOT lanes is $D_{H O T^{*}}^{L O V}$ and thereby dwindles the arrival curve, as represented by the dotted, red line. The reduced horizontal distance that results is the updated $T_{G P}$.

\subsection{Bi-Level Framework for Toll Pricing}

As toll price is imposed, some LOVs change from GP to HOT lanes, thereby reducing the demand in GP lanes. As a result, travel time in GP lanes diminishes. Due to this change, actual travel time savings experienced by HOT users become smaller than that which they expected as they entered the HOT lane. Therefore, if the posted toll price is determined based purely on the current travel time and demand, then HOT users would overpay for travel time savings. To reflect this, the proposed pricing method uses a bi-level approach for toll pricing. The upper-level described in Section 1.2 is used to determine toll price for given input values. At this level, toll price is determined based on input demand and travel time. Since demand and travel time change as the toll is imposed, it is necessary to reflect these changes. The lowerlevel updates the input values of demand and travel time by using the algorithmic steps provided in Section 1.3.

This procedure is initiated by determining the price $P$, based on the measured demand and travel time by equations (3) and (4) at the upper-level. $P$ is then an input to the lower-level to evaluate the effects of $P$ on demand and travel time. This returns updated demand and travel time. The updated demand and travel time are entered to the upper-level procedure in order to update $P$. This process is repeated until the variables - demand, travel time and price - have the same values as they had in the prior iteration.

\section{Case Study}

This section describes simulation experiments that were designed to replicate the situations that exist when a HOV lane is converted to a HOT lane without making any other changes. Traffic data from the HOV lane on the Gyungbu expressway were obtained from the Korea Expressway Corporation, and the drivers' behavioural data were estimated based on the survey data.

\subsection{Site Description}

\subsubsection{HOV Lane on the Gyungbu Expressway in South Korea}

The Gyungbu expressway in South Korea extends 416 $\mathrm{km}$, connecting Seoul and Busan, two major metropoli$\tan$ cities in South Korea. Due to growing congestion on the expressway near the Seoul metropolitan area, $37.9-\mathrm{km}$ HOV lanes have been implemented as a traffic management strategy in each direction for the hours between 7:00 A.M and 9:00 P.M. There are four lanes for this section of freeway, including one HOV lane that is separated by double-striped pavement marking. The requirements for using this HOV lane are that buses and vans with capacity for nine or more passengers must be carrying at least six passengers, more details in Jang et al. (2014).

\subsubsection{Necessary Conditions for Implementing HOT Lane}

The HOT lane is a concept for improving trip efficiency on freeways with HOV lanes; however, converting the HOV lanes to HOT lanes does not always guarantee improved traffic conditions, and sometimes the conditions are degraded. The following conditions must be satisfied to avoid adverse effects. Dahlgren (2002) and Ungemah, Swisher (2006) showed that (1) recurring congestion in the GP lanes and (2) unused capacity in the HOV lane are two necessary conditions that ensure successful implementation of a HOT lane. To check these conditions, we analysed 30-sec, aggregated traffic data that were collected via inductive loop detectors installed on the expressway.

Fig. 2a shows time-series profiles of vehicle speed in the HOV and GP lanes during morning peak-traffic hours between 7:00 A.M. and 9:00 A.M. The profiles show that vehicle speed in the HOV lane is about $50 \mathrm{~km} / \mathrm{h}$ faster than that in the slow, congested GP lanes, indicating that the HOT lane, if converted from the HOV lane, can attract drivers who seek to travel faster for a fee.

Fig. $2 \mathrm{~b}$ shows flow vs. occupancy (a dimensionless measure of density, which is measured by an inductive loop detector) scatter plots in the HOV lane for the same time period. The data form a linear relation, meaning that traffic was in the free-flow regime.

The measured flow was less than 1100 vehicles per hour, and detector occupancy was below $10 \%$, indicating that the lane is being operated well under its capacity (Jang, Cassidy (2012); Jang et al. (2012) use empirical data and show that the relations between flow and occupancy in multiple HOV lanes begin bending downward at occupancies well above about $10 \%$, and accompanying flow is also well above $1000 \mathrm{veh} / \mathrm{h}$ ). The findings from Figs $2 \mathrm{a}$ and $2 \mathrm{~b}$ show that the traffic in the HOV lane on the Gyungbu expressway is qualified to be converted from the HOV lane to a HOT lane. 
a)

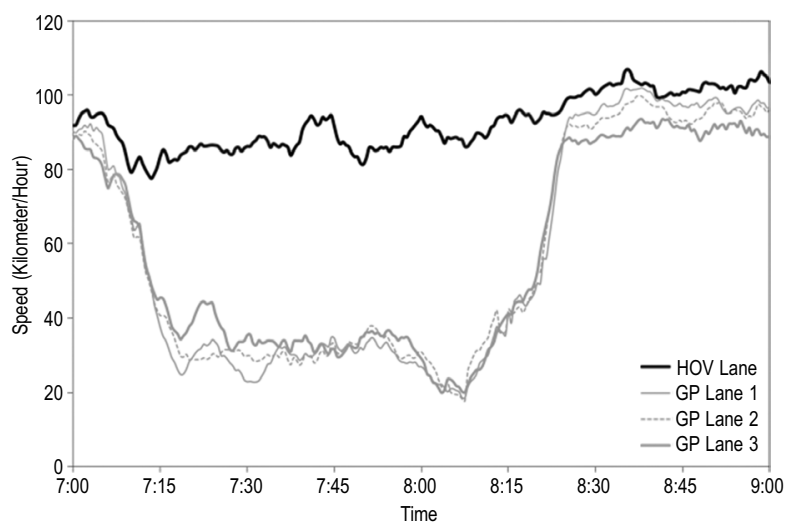

b)

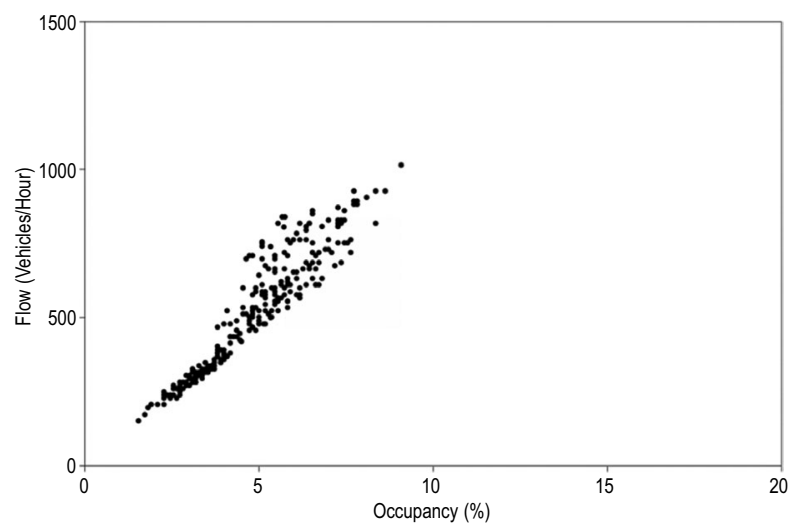

Fig. 2. Necessary conditions for HOV lane conversion to an HOT lane on the Gyungbu expressway in South Korea

(February 9, 2009): a- time-series speed profile; b - flow-occupancy plot (HOV lane)

\subsection{Drivers' Willingness to Pay}

\subsubsection{Survey Data}

To estimate the distribution of drivers' WTP, a survey was conducted in major transportation facilities (e.g., terminals and subway stations) in Seoul. Seoul was selected because potential users for the HOT lane in this study are mainly residents of Seoul and its suburban cities. Random, face-to-face interviews were conducted with commuters. The interviewer explained the survey questionnaire to each participant. Then, the respondents were asked to evaluate 15 virtual choice situations and to choose her/his preferred choice for each situation. A total of 312 respondents participated in the survey, collecting a total of 4680 responses.

The survey questionnaire was composed of two parts, one regarding general information about the survey respondents and the other regarding their travel choice, i.e., the GP lane with no fee or the HOT lane for a fee. In the former part, respondents were required to describe their socio-demographic attributes, such as age, gender, income, and household size. In the latter part, the respondents were given 15 choice situations offering two alternatives between the HOT lane and the GP lanes. The HOT lane trips have two attributes - de- creased travel time and a toll - while the GP lane trips have only one attribute, travel time. This latter part was developed based on a Stated Preference (SP) framework because it allows multiple observations on each individual's choice behaviour for situations with hypothetical attributes that do not currently exist in the market (Bateman 2004; Boxall et al. 1996; Fowkes, Wardman 1988; Hensher et al. 2005a, 2005b; Kroes, Sheldon 1988).

Values of the attributes - travel time and toll - were assigned 10 different levels that were derived based on actual measurements to realistically replicate the individual's experiences or what may appear to be plausible (Fowkes, Wardman 1988; Hensher 1994). In composing the survey questionnaire, however, we used the survey design method proposed by Van der Waerden et al. (2011). This method used a fractional, factorial, orthogonal design that selected an orthogonal fraction of full factorial design and combined choices of sets into a smaller number if the attributes could be studied independently from each other, which occurred in our case. Hence, the survey questionnaire was further reduced to 15 random choice sets.

\subsubsection{Estimated Outcome}

The data collected during the survey were analysed using the mixed logit model and the estimated outcome is summarized in a Table. In this estimation, we assumed that all drivers made their decisions based on toll price and travel time, because the attributes of drivers who were actually using the expressway were exogenous and may not have changed after implementing the HOT lanes.

The estimated coefficients for both variables were statistically significant. From the estimated mean and standard deviation of travel time and cost, we derived drivers' WTP for travel time savings, which is the ratio of the travel time coefficient to the travel cost coefficient. Since the coefficients for travel time and cost are specified as log-normal distributions, the ratio also follows a log-normal distribution (see Jang et al. (2014) for more details.) Thus, the distribution of the estimated drivers' WTP for travel time saving shown in Fig. 3 has a median of 9.57 and a mean of 11.07 (the median of $\$ 9.57 / \mathrm{h}$ and a mean of $\$ 11.07 / \mathrm{h}$ can be converted to $10527 \mathrm{KRW} / \mathrm{h}$ and $12177 \mathrm{KRW} / \mathrm{h}$, respectively (the currency rate is $1100 \mathrm{KRW} / \$)$ ). These numbers are within the reasonable range of previously reported measures of travellers'

Table. Mixed logit model of expressway lane choice

\begin{tabular}{llll}
\hline \multicolumn{1}{c}{ Variable } & \multicolumn{1}{c}{ Parameter } & Value & Std. error \\
\hline \begin{tabular}{l} 
Toll $\begin{array}{l}\text { price (\$) } \\
\text { pyyy }\end{array}$ \\
\cline { 2 - 4 }
\end{tabular} & Mean of ln (-coefficient) & 0.4282 & 0.1235 \\
\hline $\begin{array}{l}\text { Travel dev. of ln (-coefficient) } \\
\text { time (h) }\end{array}$ & 0.7940 & 0.1086 \\
\cline { 2 - 4 } & Mean of ln (-coefficient) & 1.8311 & 0.1903 \\
\cline { 2 - 4 } & Std. dev. of ln (-coefficient) & 0.4282 & 0.1241 \\
\hline Value of log-likelihood function $=-1561.56$ &
\end{tabular}

Value of log-likelihood function $=-1561.56$ 
a)

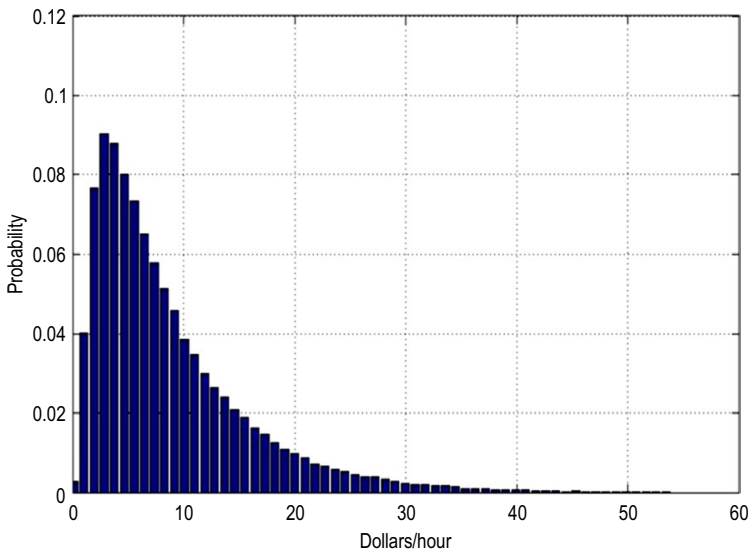

b)

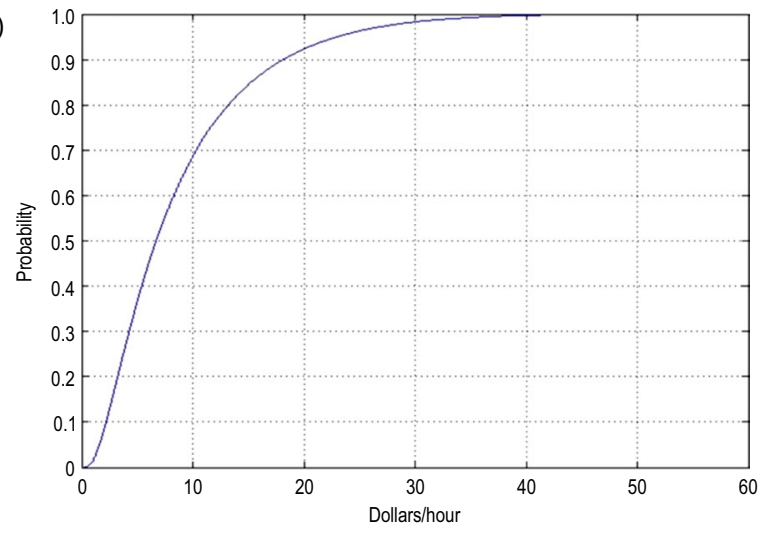

Fig. 3. Estimated distribution of drivers' willingness to pay (units: $\$ / \mathrm{h}$ ): $\mathrm{a}$ - probability density function; $\mathrm{b}$ - cumulative density function

value of time in Seoul (KDI 1999; Kim et al. 2003), and have values comparable to previously estimated values of travel time based on a different survey (Jang et al. 2014). These results indicate that the estimated drivers' WTP for travel time savings closely represents drivers' behaviours in Seoul, and the results are accurate enough to use for demonstration purposes in this study.

\subsection{Computational Simulation}

This section describes a computational simulation to demonstrate the applicability of the algorithm developed in this study. For this simulation, it was assumed that the demands of the HOV lane and the GP lanes were invariant within each time step in the simulation. The demands used in this study were those that we measured at the upstream entrance to the section of the freeway studied. The time steps in the simulation were set as five minutes; therefore, the toll price was updated every five minutes. The run time for the simulation was during the morning's peak-traffic hours, i.e., from 7:00 A.M. to 9:00 A.M.

As a first step, we computed the available capacity in the HOT lane and the excess demand for the GP lanes, because these were the necessary conditions for operating HOT lanes. Fig. 4 shows that both conditions were satisfied most of the time. Although there was no excess demand for GP lanes at times, some excess

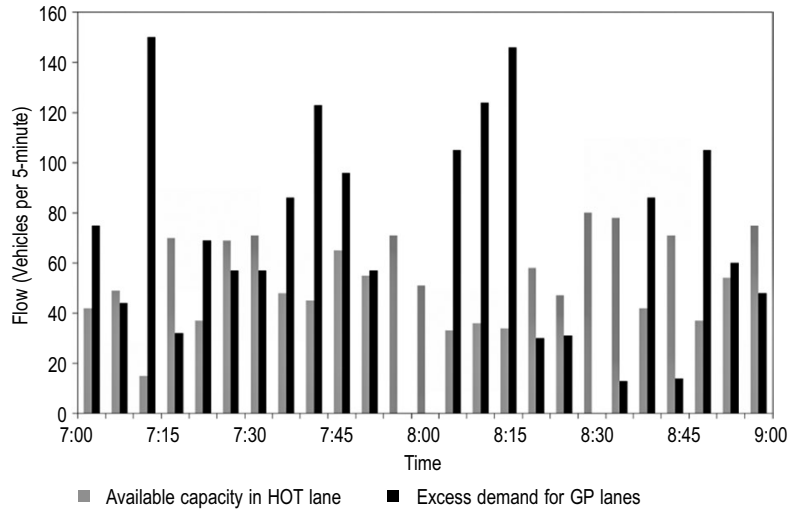

Fig. 4. Excess demand for GP lanes and available capacity in HOT lane

demand was left over from previous time steps and remained in the system, because the excess demand for GP lanes accumulates over time.

The algorithm results in higher toll prices as excess demand for GP lanes increases or available capacity in the HOT lane diminishes. As shown in Fig. 5, higher toll prices tend to correlate with higher revenue, which is the product of toll price and HOT demand. A higher toll price is set when there is higher excess demand for the HOT lane and there is some limited available capacity in the HOT lane. Therefore, the algorithm can draw as much demand as possible from the GP lane at a higher price, resulting in higher revenue. This shows that the proposed algorithm can generate some revenue by shifting demand from GP to HOT lanes.

The algorithm also reduces total travel time that is spent by drivers in the GP lanes. Drivers who had higher WTP shifted from the GP to HOT lanes, thereby reducing demand in the GP lanes. The number of vehicles that shifted to the HOT lane still remains less than the unused capacity of the HOT lane; therefore, the vehicles that shifted from GP to HOT lanes do not affect the speed of the vehicles in the HOT lane. This sequence is more pronounced during the severely congested period from 7:15 A.M. to 8:15 A.M., indicating that the proposed algorithm could be more effective, i.e., reducing the total travel time to a greater extent, when the congestion is more severe, i.e., when drivers are delayed for a longer duration (Fig. 6).

The present algorithm searches iteratively for the optimal number of shifted vehicles until the solution converges to equilibrium, because the shifting of vehicles from GP to HOT lanes enhances travel conditions in the GP lanes and, consequently, changes the optimal solution. If the algorithm computes toll price without considering this interactive behaviour between drivers and travel conditions, the algorithm tends to shift a smaller number of vehicles because it overestimates the travel time difference between GP and HOT lanes and, as a result, sets a higher toll price.

To examine the difference between considering and not considering the interactive behaviour, the numbers of shifted vehicles were computed for both cases 
a)

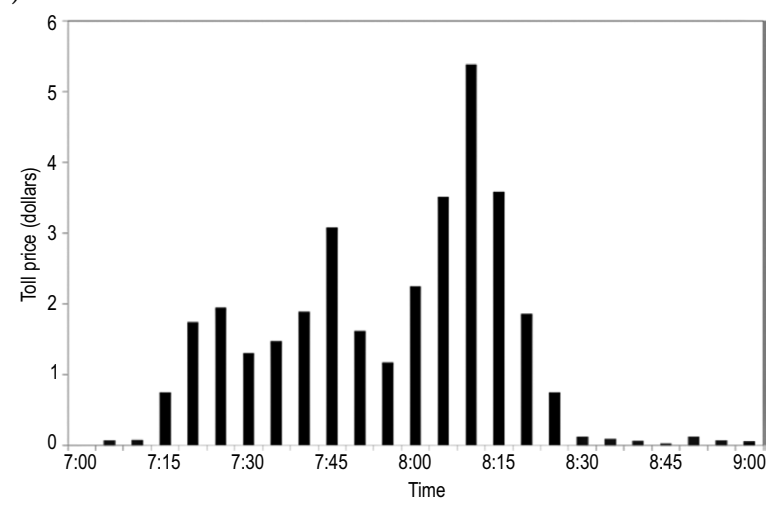

b)

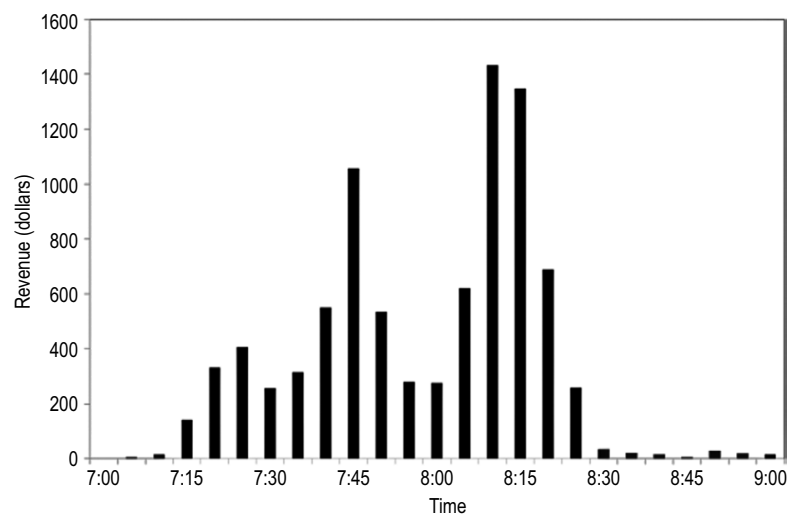

Fig. 5. Simulated (a) toll price and (b) revenue

and compared in Fig. 7. The solid black line in Fig. 7 represents the target number of vehicles that should be shifted from GP lanes to a HOT lane, which is the available capacity in the HOT lane. The solid grey line is the number of shifted vehicles that are computed when interactive behaviour is considered (i.e., iterative solution), and the dotted grey line is the number of shifted vehicles that were computed without considering the interactive behaviour (i.e., initial solution). Fig. 7 shows that the algorithm that considered interactive behaviour shifted enough demand from the GP lanes to a HOT lane to fill the unused capacity in the HOT lane.

\section{Conclusions}

HOV lanes have been implemented to allow vehicles with more passengers to bypass congested freeway sections. Due to this intrinsic set-up, HOV lanes are often operated under capacity while adjoining GP lanes are congested. The unused capacity in the HOV lane can be viewed as a waste of infrastructure resource that could otherwise be used by other travellers. To utilize better this unused capacity, the HOT lane concept has been introduced, in which drivers of LOVs would be allowed to use the lane for a fee. To operate the HOT lane effectively, however, it is essential to impose an appropriate toll that can shift the right amount of demand from the congested GP lanes to the underused HOV lane.

In this paper, we report our development of an algorithm that can be used to compute the amount of

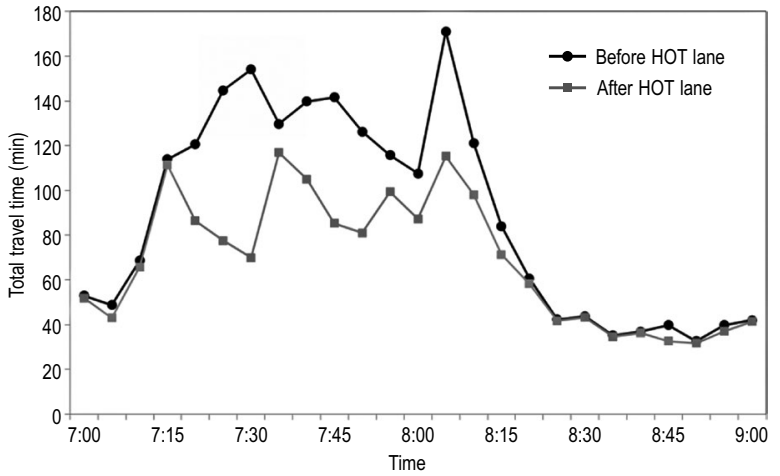

Fig. 6. Total travel time before and after HOT lane

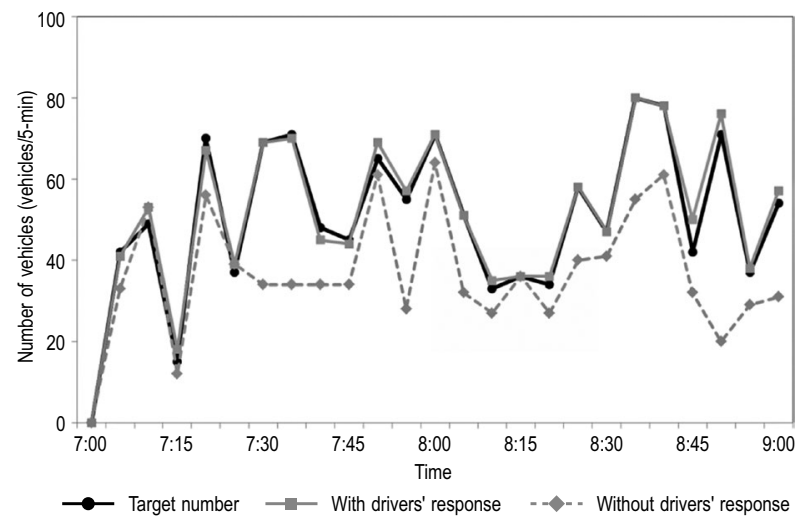

Fig. 7. Number of vehicles shifted from GP lanes to a HOT lane

toll that can attract the demand so as to minimize total travel time. The algorithm was constructed based on Stackelberg game theory in which the operator and drivers are considered to act as a leader and followers, respectively. The approach is formulated as a bi-level program, i.e., the upper-level searches for the toll price that would minimize total travel time, and the lowerlevel adjusts demand and travel time in light of drivers' responses to the toll price.

To demonstrate the applicability of this algorithm, computational simulations were performed for the scenario of converting HOV to HOT lanes on the Gyungbu expressway in South Korea. For the simulations, all the input variables (e.g., HOV and LOV traffic demand) and traffic parameters (e.g., capacity, free-flow travel time, and distribution of drivers' willingness to pay) were estimated based on real-world traffic and survey data. Given these inputs, the simulation replicated the situation in which the HOV lane was converted to a HOT lane while holding all else equal, and the toll for the HOT lane was computed using the algorithm that we developed and reported in this paper.

The outcomes of the computational simulation show that the algorithm we developed can reflect the interactions more realistically between drivers' responses and traffic dynamics that are created by the imposition of a toll. However, the algorithm is dependent upon accuracy of input data, especially varying traffic conditions in the GP lanes. By accurately estimating traffic condi- 
tions in real time, further improvements can be made. Also, the method used to obtain a reliable estimate of freeway travel time should be examined to determine the robustness of the algorithm.

\section{Acknowledgements}

This work was partially supported by the National Research Foundation of Korea grant funded by the Korea government (MEST) (NRF-2010-0029446).

\section{References}

Amador, F. J.; González, R. M.; Ortúzar, J. D. 2005. Preference heterogeneity and willingness to pay for travel time savings, Transportation 32(6): 627-647. http://dx.doi.org/10.1007/s11116-005-3734-y

Bateman, I. J.; Carson, R. T.; Day, B.; Hanemann, N.; Hett, T.; Hanley, N.; Jones-Lee, M.; Loomes, G.; Mourato, S.; Ozdemiroglu, E. 2004. Economic Valuation with Stated Preference Techniques: A Manual. Edward Elgar Publishing Ltd. $480 \mathrm{p}$.

Bhat, C. R. 2001. Quasi-random maximum simulated likelihood estimation of the mixed multinomial logit model, Transportation Research Part B: Methodological 35(7): 677693. http://dx.doi.org/10.1016/S0191-2615(00)00014-X

Boriboonsomsin, K.; Barth, M. 2007. Evaluating air quality benefits of freeway high-occupancy vehicle lanes in Southern California, Transportation Research Record 2011: 137147. http://dx.doi.org/10.3141/2011-15

Boxall, P. C.; Adamowicz, W. L.; Swait, J.; Williams, M.; Louviere, J. 1996. A comparison of stated preference methods for environmental valuation, Ecological Economics 18(3): 243-253. http://dx.doi.org/10.1016/0921-8009(96)00039-0

Brownstone, D.; Ghosh, A.; Golob, T. F.; Kazimi, C.; Amelsfort, D. V. 2003. Drivers' willingness-to-pay to reduce travel time: evidence from the San Diego I-15 congestion pricing project, Transportation Research Part A: Policy and Practice 37(4): 373-387.

http://dx.doi.org/10.1016/S0965-8564(02)00021-6

Brownstone, D.; Small, K. A. 2005. Valuing time and reliability: assessing the evidence from road pricing demonstrations, Transportation Research Part A: Policy and Practice 39(4): 279-293. http://dx.doi.org/10.1016/j.tra.2004.11.001

Burris, M. W.; Appiah, J. 2004. Examination of Houston's QuickRide participants by frequency of QuickRide usage, Transportation Research Record 1864: 22-30. http://dx.doi.org/10.3141/1864-04

Burris, M.; Lipnicky, K. 2009. HOV or general purpose lanes?, Public Works Management and Policy 14(2): 130-147. http://dx.doi.org/10.1177/1087724X09350227

Burris, M. W.; Stockton, B. R. 2004. HOT lanes in Houston six years of experience, Journal of Public Transportation 7(3): $1-21$.

Cassidy, M. J. 1998. Bivariate relations in nearly stationary highway traffic, Transportation Research Part B: Methodological 32(1): 49-59.

http://dx.doi.org/10.1016/S0191-2615(97)00012-X

Chen, C.; Varaiya, P. 2005. An empirical assessment of traffic operations, in Mahmassani, H. S. (Eds.). Transportation and Traffic Theory. Flow, Dynamics and Human Interaction. 16th International Symposium on Transportation and Traffic Theory. 19-25 July 2005, College Park Maryland, United States, 105-123.
Chung, Y.; Choi, K. 2010. Dynamic toll concept to assess feasibility of high-occupancy vehicle lane on Kyungbu freeway, South Korea, Transportation Research Record 2178: 162-169. http://dx.doi.org/10.3141/2178-18

Dahlgren, J. 2002. High-occupancy/toll lanes: where should they be implemented?, Transportation Research Part A: Policy and Practice 36(3): 239-255. http://dx.doi.org/10.1016/S0965-8564(00)00047-1

Dahlgren, J. 1998. High occupancy vehicle lanes: Not always more effective than general purpose lanes, Transportation Research Part A: Policy and Practice 32(2): 99-114. http://dx.doi.org/10.1016/S0965-8564(97)00021-9

De Palma, A.; Lindsey, R. 2011. Traffic congestion pricing methodologies and technologies, Transportation Research Part C: Emerging Technologies 19(6): 1377-1399. http://dx.doi.org/10.1016/j.trc.2011.02.010

De Palma, A.; Lindsey, R. 2004. Congestion pricing with heterogeneous travelers: a general-equilibrium welfare analysis, Networks and Spatial Economics 4(2): 135-160. http://dx.doi.org/10.1023/B:NETS.0000027770.27906.82

Fisk, C. S. 1984. Game theory and transportation systems modelling, Transportation Research Part B: Methodological 18(4-5): 301-313. http://dx.doi.org/10.1016/0191-2615(84)90013-4

Fowkes, T.; Wardman, M. 1988. The design of stated preference travel choice experiments: with special reference to interpersonal taste variations, Journal of Transport Economics and Policy 22(1): 27-44.

Hensher, D. A. 2001. The valuation of commuter travel time savings for car drivers: evaluating alternative model specifications, Transportation 28(2): 101-118. http://dx.doi.org/10.1023/A:1010302117979

Hensher, D. A. 1994. Stated preference analysis of travel choices: the state of practice, Transportation 21(2): 107-133. http://dx.doi.org/10.1007/BF01098788

Hensher, D. A.; Greene, W. H. 2011. Valuation of travel time savings in WTP and preference space in the presence of taste and scale heterogeneity, Journal of Transport Economics and Policy 45(3): 505-525.

Hensher, D. A.; Greene, W. H. 2003. The mixed logit model: the state of practice, Transportation 30(2): 133-176. http://dx.doi.org/10.1023/A:1022558715350

Hensher, D. A.; Rose, J. M.; Greene, W. H. 2005a. Applied Choice Analysis: A Primer. Cambridge University Press. $744 \mathrm{p}$.

Hensher, D.; Shore, N.; Train, K. 2005b. Households' willingness to pay for water service attributes, Environmental and Resource Economics 32(4): 509-531. http://dx.doi.org/10.1007/s10640-005-7686-7

Hess, S.; Bierlaire, M.; Polak, J. W. 2005. Estimation of value of travel-time savings using mixed logit models, Transportation Research Part A: Policy and Practice 39(2-3): 221-236. http://dx.doi.org/10.1016/j.tra.2004.09.007

Jang, K.; Cassidy, M. J. 2012. Dual influences on vehicle speed in special-use lanes and critique of US regulation, Transportation Research Part A: Policy and Practice 46(7): 11081123. http://dx.doi.org/10.1016/j.tra.2012.01.008

Jang, K.; Chung, K.; Yeo, H. 2014. A dynamic pricing strategy for high occupancy toll lanes, Transportation Research Part A: Policy and Practice 67: 69-80. http://dx.doi.org/10.1016/j.tra.2014.05.009

Jang, K.; Kim, D.; Chung, S. 2014. Heterogeneous perception of travelers on greenhouse gas pricing in Seoul, Korea, International Journal of Sustainable Transportation 8(4): 281-296. http://dx.doi.org/10.1080/15568318.2012.665981 
Jang, K.; Oum, S.; Chen, C.-Y. 2012. Traffic characteristics of high-occupancy vehicle facilities: comparison of contiguous and buffer-separated lanes, Transportation Research Record 2278: 180-193. http://dx.doi.org/10.3141/2278-20

KDI. 1999. Guideline for Pre-Feasibility Study of Road and Railway Projects. Korea Development Institute (KDI).

Kim, T.; Lim, Y.; Lee, H.; Kho, Y.; Yang, I.; Ryu, K. 2003. Value of Travel Time for Toll Roads-Seoul Metropolitan Area. Korea Research Institute for Human Settlements.

Kroes, E. P.; Sheldon, R. J. 1988. Stated preference methods: an introduction, Journal of Transport Economics and Policy 22(1): 11-25.

Kwon, J.; Varaiya, P. 2008. Effectiveness of California's high occupancy vehicle (HOV) system, Transportation Research Part C: Emerging Technologies 16(1): 98-115. http://dx.doi.org/10.1016/j.trc.2007.06.008

Li, J.; Embry, P.; Mattingly, S. P.; Sadabadi, K. F.; Rasmidatta, I.; Burris, M. W. 2007. Who chooses to carpool and why?: examination of Texas carpoolers, Transportation Research Record 2021: 110-117. http://dx.doi.org/10.3141/2021-13

Mannering, F. L.; Hamed, M. M. 1990. Commuter welfare approach to high occupancy vehicle lane evaluation: an exploratory analysis, Transportation Research Part A: General 24(5): 371-379. http://dx.doi.org/10.1016/0191-2607(90)90049-C

Poole, R. W.; Orski, C. K. 2000. HOT lanes: a better way to attack urban highway congestion, Regulation 23(1): 15-20.

Small, K. A.; Winston, C.; Yan, J. 2006. Differentiated road pricing, express lanes, and carpools: exploiting heterogeneous preferences in policy design, Brookings-Wharton Papers on Urban Affairs 7: 53-96.

Small, K. A.; Yan, J. 2001. The value of "value pricing" of roads: second-best pricing and product differentiation, Journal of Urban Economics 49(2): 310-336. http://dx.doi.org/10.1006/juec.2000.2195

Supernak, J.; Steffey, D.; Kaschade, C. 2003. Dynamic Value pricing as instrument for better utilization of high-occupancy toll lanes: San Diego I-15 case, Transportation Research Record 1839: 55-64. http://dx.doi.org/10.3141/1839-05

Train, K. E. 2009. Discrete Choice Methods with Simulation. 2nd edition. Cambridge University Press. 408 p.

Train, K. 1999. Halton Sequences for Mixed Logit. Working paper. Department of Economics, University of California, Berkeley. 18 p. Available from Internet: http://eml.berkeley. edu/wp/train0899.pdf

Train, K.; Sonnier, G. 2005. Mixed logit with bounded distributions of correlated partworths, in Scarpa, R.; Alberini, A. (Eds.). The Economics of Non-Market Goods and Resources. Vol. 6: Applications of Simulation Methods in Environmental and Resource Economics, 117-134.

http://dx.doi.org/10.1007/1-4020-3684-1_7

Ungemah, D.; Swisher, M. 2006. So you want to make a highoccupancy toll lane?: Project manager's guide for conversion from high-occupancy vehicle lane to high-occupancy toll lane, Transportation Research Record 1960: 94-98. http://dx.doi.org/10.3141/1960-12

Van der Waerden, P.; De Bruin, M.; Timmermans, H.; Van Loon, P. 2011. Travelers' willingness to use park and ride facilities and additional transport in the context of commuting and shopping trips to city centers, in TRB 90th Annual Meeting Compendium of Papers DVD, 23-27 January 2011, Washington, D.C. 13 p. (DVD)
Windover, J. R.; Cassidy, M. J. 2001. Some observed details of freeway traffic evolution, Transportation Research Part A: Policy and Practice 35(10): 881-894. http://dx.doi.org/10.1016/S0965-8564(00)00029-X

Yin, Y.; Lou, Y. 2009. Dynamic tolling strategies for managed lanes, Journal of Transportation Engineering 135(2): 45-52. http://dx.doi.org/10.1061/(ASCE)0733-947X(2009)135:2(45)

Zhang, H. M.; Ge, Y. E. 2004. Modeling variable demand equilibrium under second-best road pricing, Transportation Research Part B: Methodological 38(8): 733-749. http://dx.doi.org/10.1016/j.trb.2003.12.001 